\title{
Análisis bibliométrico y de mapeo de la educación en cirugía laparoscópica y robótica en urología
}

\section{Bibliometric Analysis of Education in Laparoscopic and Robotic Surgery in Urology}

\author{
Lynda Torres $^{1}$ Adriana Pedraza $^{2}$ Lisset Arroyo $^{3}$ Melanie López de Mesa ${ }^{4}$ Verónica Tobar ${ }^{5}$ \\ Pamela Perdomo ${ }^{6}$ Ana Maria Cárdenas ${ }^{7}$ Catalina Solano ${ }^{8}$
}

${ }^{1}$ Médica Uróloga, Centro Policlínico Olaya, Bogotá, Colombia

2 Médica Uróloga, Clínica Desa, Cali, Colombia

3 Médica Uróloga, Hospital Simón Bolívar, Bogotá, Colombia

${ }^{4}$ Médica Uróloga, Hospital San José, Clínica de Marly, Bogotá, Colombia

${ }^{5}$ Médica Uróloga, Clínica Foscal, Bucaramanga, Colombia

${ }^{6}$ Médica Uróloga, Clínica Antioquia, Medellín, Colombia

${ }^{7}$ Médica Uróloga, Clínica Nueva El Lago, Bogotá, Colombia

8 Médica Uróloga, Uroclin, Medellín, Colombia
Address for correspondence Lynda Torres, Correspondencia a la carrera 7 \# 40-62 (e-mail: lynda.torresc@gmail.com).

\begin{abstract}
Resumen
Palabras clave

- bibliométrico

- laparoscopia

- educación

- urología

Objetivo Se realizó un análisis bibliométrico para conocer la situación actual de la educación en cirugía laparoscópica y robótica en urología.

Métodos La búsqueda fue realizada en la base de datos MEDLINE y EMBASE en marzo de 2020, a través del facilitador de búsqueda: FABUMED y PUBMED-PUBREMINER, utilizando los siguientes criterios (Laparoscopy OR robotics) AND (education urology) AND (1988:2019[dp])) y establecer los artículos disponibles respecto a educación en cirugía laparoscópica en urología. Adicionalmente se realizó un análisis de mapeo bibliométrico así como mapas de redes de colaboración.

Resultados Se obtuvieron 1383 revisiones en la literatura publicadas en 172 revistas. Las revistas con más publicaciones fueron: Journal of Endourology (167) y factor de impacto (FI) 2.26, BJU International (101, FI 4.52) Urology (90, FI 1.86) y Journal of Urology (71, Fl 5.6). La producción total se dio en 49 países, los países con mayor número de publicaciones fueron Estados Unidos con 362 (26.1\%) y Reino Unido 152 (10.9\%), Latinoamérica estuvo encabezada por Brasil con 17 publicaciones.

Conclusiones El mayor número de publicaciones se observó a partir del 2006 years durante la última década ha estado liderado por Estados Unidos. En Colombia la investigación de educación en cirugía laparoscópica y robótica es mínima, posiblemente por carecer de programas de entrenamiento en los programas de residencia. Este análisis bibliométrico permite mostrar que la producción bibliográfica en esta área puede depender de que existan programas formales de entrenamiento que a futuro beneficien a toda la comunidad urológica.
\end{abstract}

received

August 30, 2020

accepted

October 27, 2020
DOI https://doi.org/

10.1055/s-0040-1721331.

ISSN 0120-789X.

e ISSN 2027-0119.
Copyright ( $\odot$ 2020, Thieme Revinter Publicações Ltda.., Rua do Matoso 170, Rio de Janeiro, RJ, CEP 20270-135, Brazil. Todos los derechos reservados.
License terms

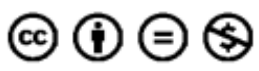


Abstract

\section{Keywords}

- bibliometrics

- laparoscopy

- education

- urology
Objective A bibliomeric analysis was performed to know the current situation of education in laparoscopic and robotic surgery in Urology.

Methods The literature search was made using MEDLINE and EMBASE database on March 2020, we used de search facilitators FABUMED and PUBMED-PUBREMINER, we used the following criteria por the search (Laparoscopy OR robotics) AND (education urology) AND (1988: 2019 [dp])) and we obtained the articles available regarding education in laparoscopic surgery in urology. Additionally, a bibliometric mapping analysis was performed as well as collaboration network maps.

Results We found 1383 reviews in the literature published in 172 journals. The journals with most publications were: Journal of Endourology (167) and impact factor (FI) 2.26, BJU International (101, FI 4.52) Urology (90, FI 1.86) and Journal of Urology (71, Fl 5.6). Overall production was in 49 countries, the countries with the highest number of publications were the United States with $362(26.1 \%)$ and the United Kingdom 152 (10.9\%), Latin America was led by Brazil with 17 publications.

Conclusions Most of publications was observed from 2006 and during the last decade it has been led by the United States. In Colombia, educational research in laparoscopic and robotic surgery is minimal, possibly due to the lack of training programs in residency programs. This bibliometric analysis shows that bibliographic production in this area may depend on the existence of formal training programs that will benefit the entire urological community in the future.

\section{Introducción}

En el ámbito quirúrgico urológico la cirugía mínimamente invasiva toma un rol cada vez más importante, hasta el punto de convertirse en el estándar de oro actual para ciertos procedimientos. ${ }^{1}$

Por este motivo, las herramientas de aprendizaje tradicional en cirugía han ido cambiando en los últimos años, buscando que los urólogos en formación logren adquirir las capacidades necesarias para dominar adecuadamente estas técnicas. ${ }^{1-4}$

El modelo tradicional de enseñanza ha ido variando, enfocándose ahora en estrategias de aprendizaje basadas en competencias y simulación. ${ }^{5}$ Este nuevo modelo involucra conocimiento teórico y desarrollo de habilidades técnicas diferentes a las necesarias para cirugía abierta, tales como la coordinación entre el ojo y los movimientos de la mano, la visión bidimensional y la falta de háptica, entre otros. ${ }^{6}$ Los simuladores permiten desarrollar y mejorar las destrezas en el escenario de cirugía mínimamente invasiva, lo que se traduce en prácticas más seguras que reducen las tasas de morbimortalidad y mejoran el rendimiento del cirujano. ${ }^{7-10}$

Algunos estudios realizados en escuelas de cirugía general han demostrado que los residentes muestran deficiencias en su entrenamiento en cirugía laparoscópica y robótica. ${ }^{1}$ En cirugía urológica laparoscópica y robótica es importante definir las herramientas de enseñanza y los modelos de aprendizaje, compararlos y aclarar cuáles son las competencias que debe adquirir un residente en entrenamiento, así como la forma de evaluar dichas habilidades. ${ }^{11-15}$
Teniendo en cuenta la importancia que tiene el aprendizaje de la cirugía laparoscópica y robótica, se realizó un análisis bibliométrico y de mapeo para conocer la situación actual de la educación en cirugía laparoscópica y robótica en urología.

\section{Métodos}

Se realizó la búsqueda en la base de datos MEDLINE y EMBASE con literatura disponible hasta marzo de 2020, a través de la nueva herramienta FABUMED y PubMed, utilizando los siguientes criterios de búsqueda ((Laparoscopy OR robotics) AND education urology)) AND (1988:2019[dp])).

Se realizó un análisis bibliométrico descriptivo y retrospectivo de la literatura médica disponible que nos permite medir y visualizar la influencia de los resultados de la revisión para la comunidad científica. Se incluyeron artículos de revista, artículos originales de investigación, reseñas, ensayos y otros informes científicos, con el fin de analizar toda la literatura médica indexada en relación con este tema. Se asumió la dirección del primer autor como origen del documento debido a que PubMed no publica información adicional.

Para el análisis de los datos se utilizaron las herramientas estadísticas de FABUMED y PubReminer para la obtención la información de las variables a analizar, se tuvieron en cuenta datos como el número total de publicaciones y revistas, número y porcentaje de publicaciones por revista y país, patrón de producción científica a lo largo del período 
estudiado, instituciones involucradas en la producción científica, número de autores, artículos más citados. Adicionalmente realizamos un análisis de mapeo bibliométrico utilizando el programa de software: VOSviewer que explora las tendencias de palabras clave y los conceptos relacionados, así como mapas de redes de colaboración.

Usando técnicas de agrupamiento ${ }^{16,17}$ se exploraron las interacciones de palabras clave y palabras clave de título y resumen de la literatura encontrada, con el fin de mapear el dominio del conocimiento científico y revelarle al lector una manera simple y rápida de ver los datos. Se adoptó el análisis de co-ocurrencia para investigar las áreas populares y direcciones de investigación y fue clave para monitorear desarrollos en áreas científicas.

Los resultados fueron analizados de forma estadística y descriptiva y con base en esta información, se elaboraron tablas y gráficas en Microsoft Office Excel 2017. A través de Journal Citation Reports 2017/2018 se obtuvo el factor de impacto (FI) de las revistas encontradas.

\section{Resultados}

Se recuperaron en total de 1383 revisiones en la literatura en un lapso de 31 años (1988 hasta 2019). -Figura 1. Los artículos fueron publicados en 172 revistas, sin embargo, el $57.9 \%$ se realizaron en las 20 primeras revistas encontradas en la - Tabla 1. Las revistas con mayor número de publicaciones fueron, el Journal of Endourología con 167, y un factor de impacto (FI) 2.26, BJU Int con 101 (FI 4.52) Urology 90 (FI 1.86) y J Urol con 71 (FI 5.6), estas 4 revistas de idioma inglés. La revista con mayor FI fue el European Urology, con 17.29. Solo 4 revistas obtuvieron un FI mayor de 3. - Tabla 1.

\section{Autores}

Se identificaron en total 4477 autores en FABUMED y PubReminer. El autor con más número de publicaciones (35) y citaciones (118) fue Dasgupta P (King's College London y MRC Centre for Transplantation and Urology Centre, London, UK). En la - Figura 2, se muestran los diez autores principales en investigación sobre esta área, con su total de citaciones.

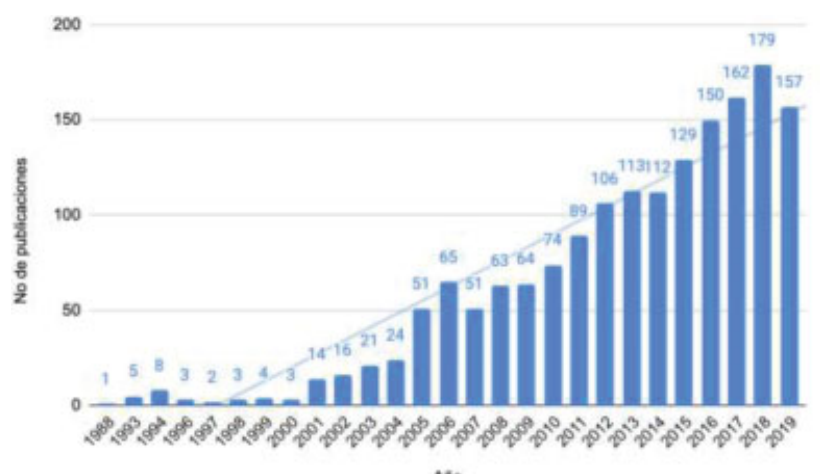

AnO

Fig. 1 Crecimiento de publicaciones globales en las últimas 3 décadas acerca de formas de educación en laparoscopia en urología.

\section{Instituciones}

La institución con mayor número de publicaciones (53) es University of California System, de USA, podemos ver en la - Tabla 2 que la mayoría de instituciones participantes pertenecen a Estados Unidos y Londres.

\section{Países}

Se encontraron publicaciones de 49 países, la mayoría de publicaciones son de Estados Unidos con 362 (26.1\%), Reino Unido 152 (10.9\%), Italia 64 (4.6\%), Alemania 60 (4.3). Latinoamérica estuvo encabezada por Brasil con 17, seguida de Argentina con 10, México y Venezuela con 3 cada uno, Colombia y Uruguay con una publicación. - Figura 3.

\section{Artículos más citados}

El artículo con mayor número de citaciones fue publicado por Ahlering, TE, con un total de 479 citaciones. Ocho de los diez artículos son provenientes de Estados Unidos, uno de Londres y uno de Países bajos. - Tabla 3.

\section{Análisis de co-ocurrencia utilizando palabras claves}

El total de los 1383 artículos fueron visualizados en VOSviewer para identificar las áreas de investigación más importantes en el tema, se analizó la co-ocurrencia entre palabras clave y palabras clave por título y resumen. El análisis de co-ocurrencia basado en palabras clave, que tuvieran un mínimo de cinco repeticiones, se demuestra en la - Figura 4a, organizados en siete grupos con un total de 366 ítems .

El Grupo 1 (rojo) está construido con las palabras clave relacionadas con las competencias clínicas que deben tener los residentes en cirugía mínimamente invasiva dentro de un currículo: procedimiento cirugía robótica, simulación por computador, entrenamiento de simulación de cirugía. El Grupo 2 (verde) describen las relaciones entre curva de aprendizaje con: resultados en el tratamiento, complicaciones, tiempo quirúrgico y el Grupo 3 (azul) la relación entre los procedimientos urológicos, con los modelos anatómico, modelos en 3D, modelos en animales.

El análisis de un tema de tendencia en línea de tiempo es una herramienta de mapeo importante que se requiere para demostrar la evolución de la literatura sobre la educación médica en cirugía laparoscópica y robótica, los temas actuales se basan entrenamiento en simulación, realidad virtual, cirugía robótica. - Figura $\mathbf{4 b}$.

\section{Análisis de co-ocurrencia entre palabras claves, Título y resumen}

En el análisis de co-ocurrencia entre palabras claves de título-resumen, se encuentran un total de 1048 palabras clave identificadas con más de cinco repeticiones; la - Tabla 4 muestra las que presentan mayor número co-ocurrencia.

La agrupación de palabras clave de co-ocurrencia también se analizó en función del tiempo medio en que aparecieron (de 2010 a 2016) en todas las publicaciones incluidas. El azul indica que las palabras clave aparecieron antes de 2010 years el amarillo después de 2018. 
Tabla 1 Listado de las 20 revistas con mayor número de publicaciones relacionados con educación en laparoscopia y robótica en urología durante el período 1988 a 2019, su factor de impacto (FI) para el año 2017-2018, el número de publicaciones, el idioma

\begin{tabular}{|l|l|l|l|l|}
\hline Revista & Artículos & Fl 2017-2018 & Idioma & País \\
\hline J Endourol & 167 & 2.26 & Inglés & Estados Unidos \\
\hline BJU Int & 101 & 4.52 & Inglés & Inglaterra \\
\hline Urology & 90 & 1.86 & Inglés & Estados Unidos \\
\hline J Urol & 71 & 5.64 & Inglés & Estados Unidos \\
\hline Eur Urol & 50 & 17.29 & Inglés & Países Bajos \\
\hline World J Urol & 42 & 2.76 & Inglés & Estados Unidos \\
\hline Surg Endosc & 41 & 3.20 & Inglés & Estados Unidos \\
\hline J Robot Surg & 38 & $*$ & Inglés & Inglaterra \\
\hline J Surg Educ & 36 & 2.20 & Inglés & Estados Unidos \\
\hline JSLS & 24 & 1.65 & Inglés & Estados Unidos \\
\hline Int J Urol & 20 & 2.10 & Inglés & Japón \\
\hline Curr Opin Urol & 18 & 2.05 & Inglés & Inglaterra \\
\hline Curr Urol Rep & 17 & 2.07 & Inglés & Estados Unidos \\
\hline J Laparoendosc Adv Surg Tech A & 16 & 1.32 & Inglés & Estados Unidos \\
\hline Actas Urol Esp & 15 & 1.13 & Español & España \\
\hline Can J Urol & 12 & 1.03 & Inglés & Canadá \\
\hline Int Braz J Urol & 12 & 1.04 & Inglés & Brasil \\
\hline Arch Esp Urol & 11 & 0.33 & Multilengua & España \\
\hline Int J Med Robot & 11 & 1.63 & Inglés & Inglaterra \\
\hline Am J Surg & 10 & 2.20 & Inglés & Estados Unidos \\
\hline
\end{tabular}

( ${ }^{*}$ no se encontró Fl para este periodo de tiempo).

\section{Discusión}

La cirugía urológica ha evolucionado dramáticamente a través de los últimos años llevando a la necesidad de optimizar el aprendizaje de nuevas tecnologías como la cirugía mínimamente invasiva. En este escenario ha sido importante la implementación de herramientas que ayudan en el desarrollo de habilidades técnicas sostenibles en el tiempo, como lo es el entrenamiento con modelos de simulación, ${ }^{18,19}$ los cuales han demostrado resultados

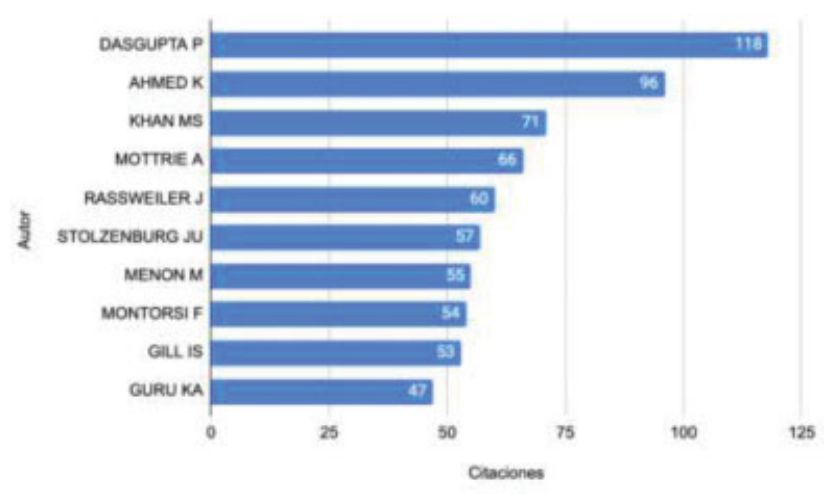

Fig. 2 Listado de los autores y sus citaciones en artículos sobre educación en laparoscopia y robótica en urología durante el período 1988 a 2019. positivos en el entrenamiento en procedimientos mínimamente invasivos.

La simulación surge como una herramienta complementaria de aprendizaje en cirugía laparoscópica, mediante el entrenamiento en un ambiente seguro, controlado y estandarizado, sin comprometer la seguridad del paciente. ${ }^{20}$ Las habilidades psicomotoras específicas y la coordinación ojo-mano necesarias para la cirugía mínimamente invasiva, se pueden dominar en gran

Tabla 2 Top 10 de las instituciones con mayor participación y número de artículos

\begin{tabular}{|l|l|}
\hline Institución & Artículos \\
\hline Universidad de California & 53 \\
\hline Fundación Guys st Thomas NHS & 38 \\
\hline Universidad de Londres & 38 \\
\hline Universidad de Irvine California & 69 \\
\hline Kings College London & 55 \\
\hline Universidad de Texas & 23 \\
\hline Cleveland Clinic & 21 \\
\hline Clinica Mayo & 36 \\
\hline Universidad Ruprecht Karls Heidelberg & 17 \\
\hline Universite de Paris & 17 \\
\hline
\end{tabular}




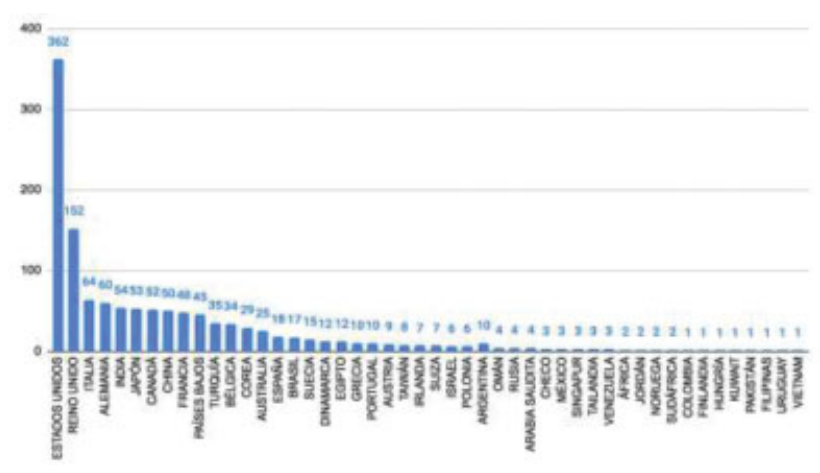

Fig. 3 Países participantes en las publicaciones 1988-2019.

medida utilizando técnicas de simulación, ${ }^{21-23}$ logrando asimismo reducción de errores, aumento en la precisión y disminución del tiempo quirúrgico. ${ }^{24-27}$

Para acceder al conocimiento y suplir las expectativas profesionales es necesario evaluar como la disponibilidad de bibliografía causa impacto en este aprendizaje. Desde nuestro punto de vista no han sido evaluadas las tendencias de publicaciones y citaciones en el aprendizaje de cirugía mínimamente invasiva. Este es el primer análisis bibliométrico realizado sobre la implementación e impacto de modelos de simulación en el proceso de aprendizaje y formación de los especialistas en cirugía mínimamente invasiva.

De acuerdo a la revisión de la literatura, Estados Unidos lidera el número de publicaciones con 362 , casi duplicando la producción bibliográfica de Reino Unido, siendo este el país europeo con mayor número de manuscritos. Este número es bajo en países de América Latina y, de éstos lidera el índice de publicaciones Brasil con 17 de ellas. Cabe resaltar que existen además artículos de México, Argentina, Colombia. Lo anterior puede ser explicado por la ausencia de implementación de subespecialización en cirugía laparoscópica o mínimamente invasiva y de sociedades científicas de dicha subespecialidad, adicionalmente, por el limitado uso de cirugía robótica en nuestro ámbito.

La mayoría de publicaciones se realizaron en revistas americanas, seguida por publicaciones europeas. La revista con mayor número de artículos es Journal of Endourology con 167, seguida de BJU International con 101 publicaciones y Urology 90 publicaciones. En total se encontraron 1383 publicaciones en la búsqueda realizada.

A partir del nuevo milenio se incrementó el número artículos, éste fenómeno podría ser explicado por la disponibilidad de tecnología, el desarrollo de programas de aprendizaje y la necesidad de incluir modelos de simulación en programas de formación en Urología.

Tabla 3 Listados de los artículos con mayor número de citaciones, autor, país, institución y citación:

\begin{tabular}{|c|c|c|c|c|c|}
\hline \# & Título & Primer autor & Institución & País & $\begin{array}{l}\text { Total de } \\
\text { citaciones }\end{array}$ \\
\hline 1 & $\begin{array}{l}\text { Successful transfer of open surgical skills to } \\
\text { a laparoscopic environment using a robotic } \\
\text { interface: Initial experience with laparoscopic } \\
\text { radical prostatectomy }\end{array}$ & Ahlering, TE & $\begin{array}{l}\text { Univ. Irvine } \\
\text { California }\end{array}$ & Estados Unidos & 479 \\
\hline 2 & $\begin{array}{l}\text { Comparison of robotic versus laparoscopic } \\
\text { skills: Is there a difference in the learning } \\
\text { curve? }\end{array}$ & Yohannes, P & Univ. Creighton & Estados Unidos & 223 \\
\hline 3 & $\begin{array}{l}\text { Robotic-assisted laparoscopic prostatectomy: } \\
\text { What is the learning curve? }\end{array}$ & Herrell, SD & Univ. Vanderbilt & Estados Unidos & 218 \\
\hline 4 & Validation of surgical simulators & $\begin{array}{l}\text { McDougall, } \\
\text { Elspeth M. }\end{array}$ & Univ. California & Estados Unidos & 184 \\
\hline 5 & $\begin{array}{l}\text { Exploring the learning curve, pathological } \\
\text { outcomes and perioperative morbidity of } \\
\text { laparoscopic partial nephrectomy performed } \\
\text { for renal mass }\end{array}$ & Link, RE & $\begin{array}{l}\text { Univ. Johns } \\
\text { Hopkins }\end{array}$ & Estados Unidos & 179 \\
\hline 6 & $\begin{array}{l}\text { Global Evaluative Assessment of Robotic Skills: } \\
\text { Validation of a Clinical Assessment Tool to } \\
\text { Measure Robotic Surgical Skills }\end{array}$ & Goh, Alvin C & $\begin{array}{l}\text { Methodist Inst } \\
\text { Technol Innovat } \\
\text { \& Educ }\end{array}$ & Estados Unidos & 151 \\
\hline 7 & $\begin{array}{l}\text { Training, Credentialing, Proctoring and } \\
\text { Medicolegal Risks of Robotic Urological } \\
\text { Surgery: Recommendations of the Society of } \\
\text { Urologic Robotic Surgeons }\end{array}$ & Zorn, Kevin C. & Univ. Chicago & Estados Unidos & 137 \\
\hline 8 & $\begin{array}{l}\text { Face, Content and Construct Validity of a Novel } \\
\text { Robotic Surgery Simulator }\end{array}$ & Hung, Andrew J. & $\begin{array}{l}\text { Univ.Southern } \\
\text { California Univ } \\
\text { So Calif }\end{array}$ & Estados Unidos & 131 \\
\hline 9 & $\begin{array}{l}\text { Current status of validation for robotic surgery } \\
\text { simulators a systematic review }\end{array}$ & $\begin{array}{l}\text { Abboudi, } \\
\text { Hamid. }\end{array}$ & $\begin{array}{l}\text { Guy's \& St } \\
\text { Thomas' NHS } \\
\text { Foundation } \\
\text { Trust Guys \& }\end{array}$ & Inglaterra & 129 \\
\hline
\end{tabular}



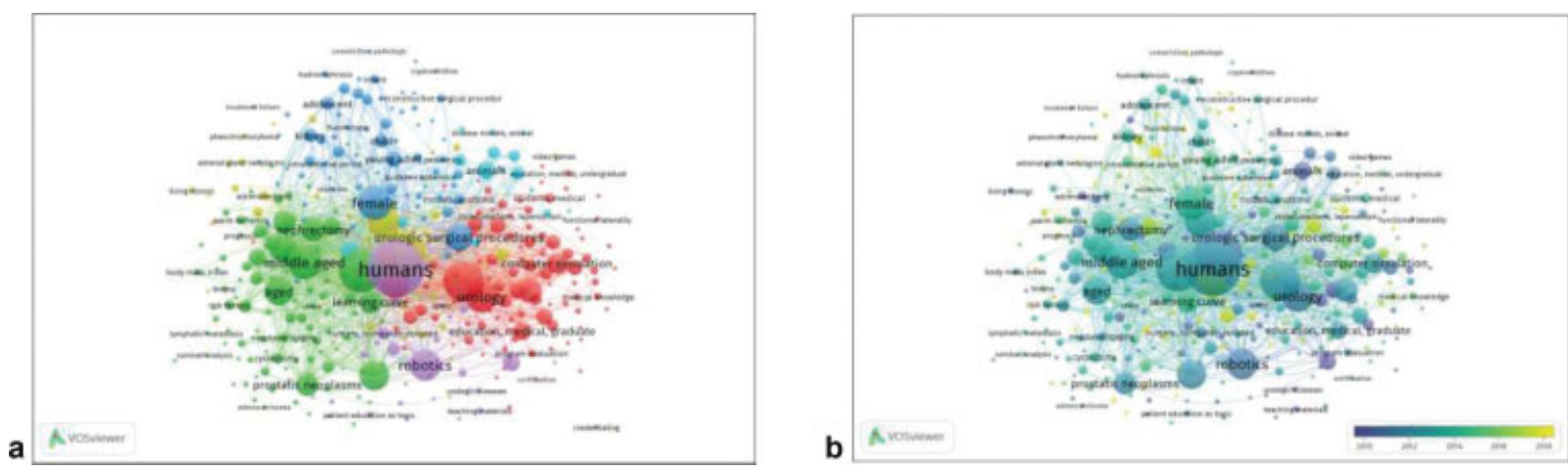

Fig. 4 Análisis de co-ocurrencia de palabras clave en los estudios de educación en cirugía mínimamente invasiva en urología. 4a Mapeo y agrupamiento de las palabras claves: - Figura 4b. Mapeo y agrupamiento de las palabras claves en línea de tiempo entre 2010-2018 (palabras en azul aparecen primero y palabras en amarillo después).

Tabla 4 Términos con mayor número de co-ocurrencia entre las palabras clave-título y resumen

\begin{tabular}{|l|l|l|}
\hline Términos & Ocurrencia & Relevancia \\
\hline Performance & 261 & 0.71 \\
\hline Complication & 219 & 0.66 \\
\hline Task & 195 & 1.18 \\
\hline Blood loss & 177 & 0.73 \\
\hline Day & 165 & 0.42 \\
\hline Operative time & 149 & 0.58 \\
\hline Simulator & 142 & 1.06 \\
\hline Expert & 138 & 0.81 \\
\hline Min & 132 & 0.81 \\
\hline Laparoscopic radical & 124 & 0.72 \\
prostatectomy & & \\
\hline Length & 117 & 0.84 \\
\hline Tool & 116 & 0.56 \\
\hline Treatment & 112 & 0.59 \\
\hline Simulation & 106 & 0.97 \\
\hline Novice & 98 & 1.18 \\
\hline Complication rate & 97 & 0.95 \\
\hline Age & 97 & 0.56 \\
\hline Validity & 96 & 1.18 \\
\hline Validation & 94 & 0.81 \\
\hline Curriculum & 92 & 1.02 \\
\hline Series & 89 & 0.55 \\
\hline Exercise & 86 & 1.23 \\
\hline Laparoscopic skill & 82 & 0.66 \\
\hline & & \\
\hline & & \\
\hline
\end{tabular}

Las principales fortalezas de nuestro estudio incluyen una amplia búsqueda de la literatura que abarca varios años para el análisis y un enfoque sistemático para identificar información relevante a través de PubMed y FABUMED.

Al ser un análisis bibliométrico existen limitaciones como el sesgo de lenguaje, las auto citaciones y el tipo de publicación. Por otro lado, PubMed registra únicamente el origen del primer autor por lo cual las colaboraciones multinacionales son indeterminadas en el presente análisis. Por último, la búsqueda por título hace que no se hayan podido identificar algunos manuscritos que tienen en su contenido temas claves del aprendizaje en cirugía mínimamente invasiva, pero que no son nombrados en el título.

A pesar de las limitaciones, nuestro estudio incluye una amplia búsqueda de la literatura para el análisis y un enfoque sistemático con el fin de identificar información relevante a través de PubMed, EMBASE, FABUMED Y VOSviewer; mostrando un resumen del panorama actual. Consideramos que será un instrumento útil para aquellos que quieran medir de manera objetiva y estandarizada el aprendizaje en cirugía mínimamente invasiva en urología.

\section{Conclusiones}

Este es el primer análisis bibliométrico realizado en Colombia que caracteriza la literatura en educación en cirugía laparoscópica y robótica, documentando que en Latinoamérica hay baja producción científica posiblemente por carecer de programas de entrenamiento formal en cirugía laparoscópica en los currículos de la residencia y que al ser incluidos podrían contribuir a futuro con la investigación urológica en esta área.

\section{Aspectos Éticos}

De acuerdo a la Resolución 8430 del año 1993 (expedida por el Ministerio de Salud de Colombia), este estudio se encuentra en una categoría sin riesgo. Se tiene además en cuenta la normatividad internacional: la declaración de Helsinki y las pautas éticas para la investigación biomédica del Consejo de Organizaciones Internacionales de las Ciencias Médicas -CIOMS- y los parámetros establecidos por la Resolución 8430 de 1993 years Resolución 2378 de 2008.

El presente estudio es descriptivo y no se realizó ningún tipo de intervención, ni modificación intencionada de variables biológicas, fisiológicas, psicológicas o sociales de los datos. Se realizó la búsqueda en la literatura para la obtención de todos los datos. 
Institucion en donde se llevó a cabo trabajo

Multi-institucional. Institución principal Uroclin.

Conflicto de intereses

Los autores declaran no tener ningún conflicto de intereses.

\section{Referencias}

1 http://www.royalcollege.ca/rcsite/cbd/competence-by-designcbd-e. Competence by Design. pp. 1-12016

2 Frank JR, Snell LS, Cate OT, et al. Competency-based medical education: theory to practice. Med Teach 2010;32(08):638-645

3 Aggarwal R, Darzi A. Technical-skills training in the 21st century. N Engl J Med 2006;355(25):2695-2696

4 Satava RM. The revolution in medical education-the role of simulation. J Grad Med Educ 2009;1(02):172-175

5 Reznick RK, MacRae H. Teaching surgical skills-changes in the wind. N Engl J Med 2006;355(25):2664-2669

6 Krummel TM. Surgical simulation and virtual reality: the coming revolution. Ann Surg 1998;228(05):635-637

7 Torkington J, Smith SG, Rees BI, Darzi A. Skill transfer from virtual reality to a real laparoscopic task. Surg Endosc 2001;15(10): 1076-1079

8 Andreatta PB, Woodrum DT, Birkmeyer JD, et al. Laparoscopic skills are improved with LapMentor training: results of a randomized, double-blinded study. Ann Surg 2006;243(06): 854-860, discussion 860-863

9 Sedlack RE, Kolars JC. Computer simulator training enhances the competency of gastroenterology fellows at colonoscopy: results of a pilot study. Am J Gastroenterol 2004;99(01):33-37

10 Grantcharov TP, Kristiansen VB, Bendix J, Bardram L, Rosenberg J, Funch-Jensen P. Randomized clinical trial of virtual reality simulation for laparoscopic skills training. Br J Surg 2004;91 (02):146-150

11 McDougall EM. Validation of surgical simulators. J Endourol 2007; 21(03):244-247

12 Van Nortwick SS, Lendvay TS, Jensen AR, Wright AS, Horvath KD, Kim S. Methodologies for establishing validity in surgical simulation studies. Surgery 2010;147(05):622-630

13 Forster JA, Browning AJ, Paul AB, Biyani CS. Surgical simulators in urological training-views of UK Training Programme Directors. BJU Int 2012;110(06):776-778
14 Vassiliou MC, Dunkin BJ, Marks JM, Fried GM. FLS and FES: comprehensive models of training and assessment. Surg Clin North Am 2010;90(03):535-558

15 Ahmed K, Amer T, Challacombe B, Jaye P, Dasgupta P, Khan MS. How to develop a simulation programme in urology. BJU Int 2011; 108(11):1698-1702

16 Van Eck NJ, Waltman L, Dekker R, Van Den Berg J. A comparison of two techniques for bibliometric mapping: multidimensional scaling and VOS. J Am Soc Inform Sci Tech 2018

17 Waltman L, van Eck NJ, Noyons ECM. A unified approach to mapping and clustering of bibliometric networks. 2010http:// arxiv.org/abs/1006.1032

18 Am Soc Inf Sci Technol. 2010;61:2405-2416

19 Academy of Medical Royal Colleges Revalidation [online]. http:// www.aomrc.org.uk/ introduction.html2009

20 Merkur S, Mossialos E, Long M, McKee M. Physician revalidation in Europe. Clin Med (Lond) 2008;8(04):371-376

21 Schreuder HW, Oei G, Maas M, Borleffs JC, Schijven MP. Implementation of simulation in surgical practice: minimally invasive surgery has taken the lead: the Dutch experience. Med Teach 2011;33(02):105-115

22 Schreuder HW, Oei SG, Maas M, et al. Implementation of simulation for training minimally invasive surgery*. TS Medisch Onderwijs 2011;30:206-220. Doi: 10.1007/s12507-011-0051-7

23 Grantcharov TP, Bardram L, Funch-Jensen P, Rosenberg J. Learning curves and impact of previous operative experience on performance on a virtual reality simulator to test laparoscopic surgical skills. Am J Surg 2003;185(02):146-149

24 Gurusamy K, Aggarwal R, Palanivelu L, Davidson BR. Systematic review of randomized controlled trials on the effectiveness of virtual reality training for laparoscopic surgery. Br J Surg 2008;95 (09):1088-1097. Doi: 10.1002/bjs.6344

25 Larsen CR, Soerensen JL, Grantcharov TP, et al. Effect of virtual reality training on laparoscopic surgery: randomised controlled trial. BMJ 2009;338:b1802. Doi: 10.1136/bmj.b1802 Erratum in: BMJ. 2009;338. doi: 10.1136/bmj.b2074

26 LeonFerrufino F, Varas CohenJ, Buckel SchaffnerE, Crovari EulufiF, Pimentel MullerF, Martınez CastilloJ, et al. Simulacion en cirugia laparoscopica. Cir Esp 2015;93:4-11

27 Lahanas V, Georgiou E, Loukas C. 2016Surgical Simulation Training Systems: Box Trainers, Virtual Reality and Augmented Reality Simula. 